\title{
AVALIAÇÃO DA CAPACIDADE ORGÁSTICA EM MULHERES NA PÓS-MENOPAUSA
}

Sonia Regina Lenharo Penteado*, Angela Maggio da fonseca, Vicente Renato Bagnoli, Joserita Serrano Assis, José Aristodemo Pinotti

Trabalho realizado no Departamento de Obstetrícia e Ginecologia do Hospital das Clínicas da Faculdade de Medicina da Universidade de São Paulo, SP

RESUMO - Ов|ETIVO. Correlacionar a capacidade orgástica (durante a relação sexual e/ou a masturbação solitária) de mulheres pósmenopausadas, saudáveis e sem tratamento hormonal com fatores climatéricos, psicossociais, comportamentais, hormonais e interpessoais.

Métodos. De um total de 999 mulheres avaliadas com idade entre $4 \mathrm{I}$ e 60 anos, selecionaram-se 60 mulheres saudáveis, sexualmente ativas, com pelo menos um ano de amenorréia, útero íntegro, relacionamento estável com parceiro capacitado ao coito e não usuárias de terapia hormonal. Elaborou-se um modelo estatístico de regressão logística que avaliou a capacidade de orgasmo (variável dependente) em função de 17 variáveis independentes, que representavam fatores psicossociais, comportamentais, interpessoais, climatéricos e hormonais.
Resultados. A capacidade orgástica está significativamente correlacionada à prática da masturbação $(p=0,000)$, ao gostar de abraçar e acariciar o corpo do parceiro $(p=0,036)$ e à presença de secura vaginal $(p=0,021)$.

Conclusöes. Nas mulheres pós-menopausadas avaliadas, a capacidade orgástica relacionou-se positivamente com o relacionamento mais afetivo com o companheiro e com a prática da masturbação. Mulheres que apresentam secura vaginal, mas que praticam a masturbação e mantêm relacionamento afetivo com o parceiro, conseguem obter o mesmo número ou um número maior de orgasmos se comparados à frequêencia do coito.

Unitermos: Pós-menopausa. Orgasmo. Masturbação.

\section{INTRODUÇÃo}

O climatério é considerado um período crítico à sexualidade por constituir a etapa onde múltiplos fatores atuam simultaneamente, de forma positiva ou negativa, favorecendo mudanças profundas. Do ponto de vista biológico, um fato relevante é que a chegada da menopausa resulta na perda do objetivo primário do sexo - a reprodução - e este fato repercutirá, com maior ou menor intensidade, no exercício da sexualidade, dependendo de aspectos psicológicos e culturais!

O estudo da sexualidade na pós-menopausa vem-se intensificando devido à maior incidência de disfunções sexuais nesta fase da vida, e também ao aumento da expectativa de vida e à predominância de mulheres na população. Muitos autores observam um declínio da atividade sexual nessa fase da vida e correlacionam este acontecimento à deficiência hormonal, uma vez que coincide com as evidências biológicas da privação estrogênica, como, por exemplo, a atrofia pélvica². O coito

*Correspondência:

Rua Capote Valente, 668 - Ap. 65 05409-002 - São Paulo - SP desconfortável ou doloroso e as eventuais contrações uterinas dolorosas durante 0 orgasmo levam algumas mulheres a evitar, sempre que possível, a experiência sexual ${ }^{3}$.

Estudos de Kinsey et $a l .^{4}$ e Masters \& Johnson ${ }^{5}$ demonstram que, dentre todas as formas de atividade sexual, a masturbação é a mais eficaz em produzir orgasmos em mulheres. Atualmente, os terapeutas sexuais prescrevem a auto-estimulação para mulheres com dificuldades sexuais; porém, observa-se que a prática da masturbação difere entre os gêneros, sendo relatada por uma taxa muito menor de mulheres do que de homens 4 .

Dentre os estudos sobre sexualidade disponíveis em bancos de dados, observa-se uma escassez no enfoque da prática de masturbação na fase do climatério ou em qualquer outra fase da vida da mulher. Considerando-se a multiplicidade e a complexidade dos fatores que influenciam a sexualidade, uma outra característica comum aos estudos que avaliam a sexualidade é o fato de utilizarem casuísticas não adequadas ${ }^{6}$.

Este estudo tem o objetivo de correlacionar a capacidade orgástica - durante a relação sexual e/ou a masturbação solitária - de mulheres pós-menopausadas, saudáveis e sem tratamento hormonal com fatores psicossociais, climatéricos, comportamentais, hormonais e interpessoais.

\section{Casuística}

No período de maio de 2000 a novembro de 2001 foram entrevistadas 999 mulheres com idade de 41 a 60 anos regularmente matriculadas no Ambulatório de Ginecologia Endócrina e Climatério da Clínica Ginecológica do Hospital das Clínicas da Faculdade de Medicina da Universidade de São Paulo.

Foram selecionadas mulheres saudáveis, menopausadas naturalmente, com pelo menos um ano de amenorréia, sexualmente ativas e com relacionamento estável com parceiro capacitado para o coito. Mulheres que apresentavam doenças sistêmicas, doenças psiquiátricas, endócrinas, distopias genitais (prolapsos uterinos do $2^{\circ}$ e $3^{\circ}$ graus e colpocistoceles do $2^{\circ}$ e $3^{\circ}$ graus) e usuárias de medicamentos que apresentavam interferência na sexualidade (tratamento hormonal, drogas anti-hipertensivas, antidepressivas, ansiolíticas, neurolépticos) foram excluídas.

Selecionou-se 60 mulheres com idade variando de 42 a 60 anos, média etária: 52, I \pm 4 anos (mediana 52). Quarenta mulheres 


\begin{tabular}{|c|c|c|}
\hline Características & $\mathrm{n}^{*}$ & $\%$ \\
\hline Sexualmente abstinentes ou com parceiro sexual incapacitado para o coito & 358 & $\overline{35,9}$ \\
\hline Sexualmente ativas, com ciclos menstruais regulares & 78 & 7,8 \\
\hline Ativas, com ciclos menstruais irregulares & 49 & 4,9 \\
\hline Ativas, histerectomizadas & 135 & 13,5 \\
\hline Ativas, não histerectomizadas, com um ano de amenorréia, usuárias de $\mathrm{TH}^{*}$ *** & 119 & 11,9 \\
\hline $\begin{array}{l}\text { Ativas, não histerectomizadas, com um ano de amenorréila, sem TH, com doenças sistêmicas } \\
\text { ou usuárias de medicamentos com interferência na sexualidade }\end{array}$ & $|3|$ & $|3|$, \\
\hline $\begin{array}{l}\text { Sem fatores de exclusão, satisfeitas sexualmente ou que não aceitaram } \\
\text { submeter-se às entrevistas sexuais }\end{array}$ & 69 & 6,9 \\
\hline Sem fatores de exclusão; concluíram a participação no estudo & 60 & 6,0 \\
\hline Total & 999 & 100,0 \\
\hline
\end{tabular}

"número de pacientes; **tratamento hormonal

\begin{tabular}{l}
\hline Quadro I - Cálculo do coeficiente de capacidade orgástica (CCO) \\
\hline$C C O=\frac{O P+O M}{F S}$ \\
$O P=$ número de orgasmos obtidos junto com o parceiro (por mês) \\
$O M=$ número de orgasmos obtidos pela masturbação solitária (por mês) \\
$F S=$ frequêencia sexual mensal
\end{tabular}

(66,7\%) eram de raça branca e 20 (33,3\%) de negra. Predominaram mulheres católicas (36), mas 16 eram evangélicas, cinco espiritualistas, duas budistas e uma delas não tinha religião. Quarenta e sete mulheres (78,3\%) haviam estudado até o primário completo, I I ( 8,3\%) até o secundário completo e duas $(3,3 \%)$ apresentavam nível superior completo. As dosagens hormonais de FSH, LH e estradiol eram compatíveis com a pós-menopausa e as dosagens de prolactina, triidotironina (T3), tiroxina (T4), tiroxina livre (T4 livre) e hormônio tireotrófico (TSH) mostraram-se dentro da faixa da normalidade.

A Tabela I mostra as características das 939 mulheres que foram excluídas do estudo.

O projeto de estudo e o termo de consentimento livre e esclarecido, foram analisados e aprovados pela Comissão de Ética para Análise de Projetos de Pesquisa (CAPPesq), em sessão de 10/05/2000, sob o Protocolo de Pesquisa n. ${ }^{\circ} 174 / 00$.

\section{Métodos}

A anamnese, o exame físico e as entrevistas sexuais foram realizados junto ao Ambulatório de Ginecologia Endócrina e Climatério do Hospital das Clínicas da Faculdade de Medicina da Universidade de São Paulo, por uma única pesquisadora e com total privacidade.

\section{Instrumentos de avaliação}

Questionário Sexual $\mathrm{HC}^{7}$ - Trata-se de questionário elaborado, inicialmente, por $\mathrm{ABDO}$ et $a l_{1}^{8}$ e ampliado no Setor de Ginecologia Endócrina e Climatério. Este questionário avalia dados demográficos, hábitos e costumes, nível de estresse, antecedentes sexuais, auto-estima e analisa, de forma detalhada, a resposta sexual.

Inventário de Satisfação Sexual - versão para mulheres (GRISS) - questionário elaborado por Golombok \& Rust ${ }^{9}$, composto de 28 perguntas que avaliam a resposta sexual e 0 relacionamento com o parceiro. As respostas variam de acordo com a intensidade, de nenhuma até a mais alta intensidade observada ou experimentada pela mulher, em relação ao questionamento realizado em cada tópico do instrumento de avaliação.

\section{A seleção das mulheres incluiu as seguintes etapas:}

Visita $n^{\circ} /$

- Anamnese, antecedentes pessoais, menstruais, sexuais; exame físico completo e exame ginecológico.
- Solicitação de exames complementares (exames laboratoriais e hormonais, ultra-som pélvico transvaginal e abdominal) para as mulheres que preenchiam, inicialmente, os critérios de inclusão e exclusão.

Visita $n^{\circ} 2$

- Avaliação dos resultados dos exames complementares. Agendada visita n. 3 para aplicação dos questionários sexuais para as mulheres que preenchiam os critérios de inclusão e exclusão.

Visita $n^{\circ} 3$

- Aplicação do Questionário sexual HC e do Inventário de Satisfação Sexual - versão para mulheres (GRISS).

\section{Capacidade orgástica}

Capacidade orgástica foi avaliada pelo coeficiente de capacidade orgástica ${ }^{7}$ (CCO). Esse coeficiente foi calculado a partir dos dados obtidos pelo questionário sexual $\mathrm{HC}$ e foi definido como a razão entre a soma do número de orgasmos obtidos com o parceiro por mês (OP) e daqueles obtidos pela masturbação, por mês (OM), e a freqüência sexual mensal (FS) (Quadro I). Foi adotado o valor de referência para o CCO igual a I, definindo-se duas classes: $\mathrm{CCO} \geq \mathrm{I}$ e $\mathrm{CCO}<1$.

\section{Fatores sociais}

Estado civil - Classificou-se as mulheres em casadas ou amasiadas (divorciadas, viúvas, solteiras, desquitadas ou separadas; todas com relacionamento sexual estável).

Renda pessoal - Definiram-se três classes de mulheres: sem renda pessoal, com renda acima da média das rendas pessoais encontradas e com renda abaixo desta média.

\section{Fatores comportamentais}

Iniciação sexual

Primeiro orgasmo - Considerou-se a fase de vida em que a mulher obteve o primeiro orgasmo: até a adolescência (idade inferior a 21 anos), ou a partir da idade adulta (idade maior ou igual a 21 anos). A avaliação da condição em que foi obtido o primeiro orgasmo, resultou na classificação das mulheres em outros dois grupos: pela masturbação solitária ou na presença de um parceiro sexual.

Primeira relação sexual - De acordo com a fase de vida em que ocorreu a primeira relação 
Penteado SRL et al.

sexual, definiram-se duas categorias: mulheres que tiveram a primeira relação sexual na adolescência ou na idade adulta. Avaliou-se, de forma detalhada, a primeira relação sexual e classificou-se essa relação em experiência positiva quando lembrada como um contato físico prazeroso, realizado em local seguro, com privacidade, com parceiro sexual afetivo, sem grau exagerado de dor ou sangramento e consentido pela mulher). A primeira relação foi considerada experiência negativa, quando citada qualquer situação oposta.

Número total de parceiros sexuais Contabilizou-se o número total de parceiros sexuais com quem a mulher manteve atividade sexual regular, por período mínimo de seis meses. Foram consideradas as mulheres que se relacionaram com um ou com dois ou mais parceiros.

Prática da masturbação - Considerou-se as mulheres que praticavama masturbação e as que não a praticavam, no momento da entrevista.

Atividade física - As mulheres foram classificadas em sedentárias ou com atividade física a depender da prática regular de exercícios com freqüência mínima de três vezes por semana.

\section{Fatores psicológicos e interpessoais}

Auto-estima - Esta avaliação foi realizada utilizando-se o questionário sexual HC. Estabeleceu-se duas classes de mulheres: com boa ou com baixa auto-estima. $O$ primeiro grupo foi composto de mulheres em que a autoavaliação demonstrou sentimentos de consideração e apreço por elas mesmas em vista de suas qualidades ou méritos. $O$ segundo grupo foi composto de mulheres que demonstravam a falta desses sentimentos.

Prazer no contato físico com o parceiro sexual - Este item foi avaliado pela pergunta: "Você gosta de abraçar e acariciar o corpo de seu parceiro?", que corresponde a uma das questões do inventário de satisfação sexual GRISS. Esta pergunta oferecia cinco opções de respostas: sempre, geralmente, ocasionalmente, quase nunca ou nunca. Foram consideradas respostas afirmativas as opções 'sempre' e 'geralmente', e negativas as opções 'nunca', 'quase nunca' e 'ocasionalmente'.

Grau de envolvimento amoroso com o parceiro sexual - Para esta avaliação utilizou-se a seguinte pergunta: "Você sente falta de amor e afeto na vida sexual com o seu parceiro?", do inventário de satisfação sexual - GRISS. Dentre as cinco opções de respostas foram consideradas afirmativas o 'sempre' e 'geralmente', e negativas o 'nunca', 'quase nunca' e 'ocasionalmente'.

\section{Parâmetros climatéricos}

Tempo de menopausa - Calculou-se a média do tempo de menopausa (em anos) e definiu-se duas classes de mulheres: acima da média encontrada e abaixo dela.

Sintomas climatéricos - Os sintomas climatéricos foram avaliados de acordo com o Índice Menopausal de Kupperman. O resultado deste índice foi considerado "leve" quando a soma dos valores atribuídos aos sintomas foi menor ou igual a 19, "moderado" quando os valores ficaram entre 20 e 35, e "acentuado" quando ele foi maior que 35 .

O aparecimento de secura vaginal e dispareunia após a menopausa permitiu o estabelecimento de dois grupos para cada parâmetro: come sem os sintomas.

\section{Fator hormonal}

As dosagens de testosterona total (ng/dl) foram realizadas pelo método de radioimunoensaio em duplicata, após a extração do soro com acetato de etila: hexana (3:2) ou hexana, sem cromatografia prévia após a extração, e depois de verificarmos que os antisoros utilizados eram específicos ${ }^{10}$. Foram considerados dois grupos de mulheres: o primeiro com dosagens séricas de testosterona total $\geq 25 \mathrm{ng} / \mathrm{dl}$ e o segundo com dosagens $<25 \mathrm{ng} / \mathrm{d}$. Segundo a experiência clínica de Kaplan", este valor adotado para se definir os dois grupos corresponde ao limite inferior da normalidade para mulheres.

\section{Influências na capacidade orgástica}

O CCO foi avaliado em função de 17 variáveis independentes, que representavam os parâmetros comportamentais, psicológicos, interpessoais, climatéricos e hormonais.

\section{Metodologia estatística}

A análise estatística foi realizada de maneira sistêmica, com o auxúlio de um modelo de regressão logística'2,13, considerando-se as seguintes variáveis:

- Variável dependente: CCO $(<\mid$ e $\geq 1)$

- Variáveis independentes: estado civil ou tipo de relação com o parceiro sexual (casada/ amasiada), renda pessoal (sem renda/ com renda até 2,8 salários mínimos/ renda acima de 2,8 salários mínimos), idade do primeiro orgasmo (até 21 anos/ acima de 21 anos), situação do primeiro orgasmo (com parceiro/ sem parceiro), faixa etária da primeira relação sexual (até 21 anos/ com mais de 21 anos), tipo de experiência representada pela primeira relação sexual (positiva/ negativa), número de parceiros sexuais (um/ dois ou mais), prática da masturbação (sim/ não), qualificação do índice de Kupperman (leve/ moderado/ acentuado), faixa da dosagem de testosterona total $(\geq 25 \mathrm{ng} / \mathrm{dl}$ ou $<25 \mathrm{ng} / \mathrm{dl}$ ), prática de atividade física (sim/não), dispareunia (sim/não), secura vaginal (sim/não), sentir falta de amor e afeto na relação sexual com o parceiro (sim/não), gostar de abraçar e acariciar o corpo do parceiro (sim/não), avaliação da auto-estima (boa/ baixa), tempo de menopausa (até seis anos/mais de seis anos).

O nível de significância do modelo ajustado e de seus parâmetros (coeficientes associados às variáveis) foi avaliado pelo teste do Qui- quadrado, utilizando-se o teste da razão de verossimilhança. Fixou-se o nível de significância em 5\%. Tabelas de contingência foram elaboradas para a análise descritiva do sentido de associação da variável independente com o CCO.

\section{Resultados}

Dentre as mulheres estudadas, 43 (71,7\%) eram casadas e 17 (28,3\%) amasiadas; dentre as últimas, quatro eram divorciadas, quatro viúvas, quatro solteiras (6,7\% cada), três desquitadas (5\%) e duas separadas (3,3\%). A média do tempo de menopausa foi de seis anos, desvio da média 5,6 anos, variação de I a 28 anos. As rendas pessoais variaram de 0,5 a 10 salários mínimos, com média de 2,8 \pm 1,9 salários mínimos (mediana 3). A média das dosagens de testosterona total foi de $23 \pm 13,1 \mathrm{ng} / \mathrm{dl}$ (mediana 17).

Duas mulheres que apresentavam anorgasmia primária foram excluídas das análises estatísticas, uma vez que se incluiu a idade do primeiro orgasmo e a situação em que ocorreu esse orgasmoentre as variáveis independentes que compuseram 0 modelo estatístico do CCO. 


\section{Tabela 2 - Modelo estatístico completo do coeficiente de capacidade orgástica - formado por todas as variáveis independentes estudadas. Distribuição e níveis das variáveis do modelo completo do coeficiente de capacidade orgástica. Teste da razão de verossimilhança para os parâmetros do modelo completo do coeficiente de capacidade orgástica}

\begin{tabular}{|c|c|c|c|c|}
\hline \multicolumn{4}{|c|}{ Coeficiente de Capacidade Orgástica; > = I $(n=28$ - 48,3\%); < I $(n=30-5 I, 7 \%)$} & \multirow[b]{2}{*}{$n$} \\
\hline Variáveis independentes & $p$ & Qui- quadrado & Níveis & \\
\hline Testosteronatotal(ng./dl) & $\overline{0,241}$ & 0,623 & $\begin{array}{c}<25 \\
>=25\end{array}$ & $\begin{array}{l}39 \\
19 \\
\end{array}$ \\
\hline Prática atividade física & 0,438 & 0,508 & $\begin{array}{l}\text { Não } \\
\text { Sim }\end{array}$ & $\begin{array}{l}39 \\
19\end{array}$ \\
\hline Idade primeira relação sexual & 0,530 & 0,466 & $\begin{array}{c}<2 \mid \text { anos } \\
>=2 I \text { anos }\end{array}$ & $\begin{array}{l}28 \\
30 \\
\end{array}$ \\
\hline Número de parceiros sexuais & 0,005 & 0,943 & $\begin{array}{c}\text { Um } \\
\text { Dois ou mais }\end{array}$ & $\begin{array}{l}31 \\
27\end{array}$ \\
\hline Sente falta de amor e afeto & 3,238 & 0,072 & $\begin{array}{l}\text { Não } \\
\text { Sim }\end{array}$ & $\begin{array}{l}35 \\
23\end{array}$ \\
\hline Gosta de abraçar/acariciar & 2,644 & 0,104 & $\begin{array}{c}\text { Não gosta } \\
\text { Gosta }\end{array}$ & $\begin{array}{l}27 \\
31\end{array}$ \\
\hline Estado civil/tipo de relação & 0,042 & 0,838 & $\begin{array}{c}\text { Amasiada } \\
\text { Casada }\end{array}$ & $\begin{array}{l}17 \\
41\end{array}$ \\
\hline$\overline{R e n d a ~ p e s s o a l ~}$ & 4,417 & 0,110 & $\begin{array}{l}\text { Semrenda } \\
<\text { média } \\
>=\text { média }\end{array}$ & $\begin{array}{l}21 \\
16 \\
21\end{array}$ \\
\hline Situação do primeiro orgasmo & 3,840 & 0,050 & $\begin{array}{l}\text { Masturbação } \\
\text { Comparceiro }\end{array}$ & $\begin{array}{l}20 \\
38\end{array}$ \\
\hline Tipo experiência I a relação sexual & 2,159 & 0,142 & $\begin{array}{l}\text { Negativa } \\
\text { Positiva }\end{array}$ & $\begin{array}{l}31 \\
27 \\
\end{array}$ \\
\hline Prática da masturbação & 13,062 & 0,000 & $\begin{array}{l}\text { Não } \\
\text { Sim }\end{array}$ & $\begin{array}{l}40 \\
18 \\
\end{array}$ \\
\hline Indice de Kupperman & 0,511 & 0,775 & $\begin{array}{c}\text { Leve } \\
\text { Moderado } \\
\text { Intenso }\end{array}$ & $\begin{array}{c}33 \\
20 \\
5\end{array}$ \\
\hline Dispareunia & 0,135 & 0,714 & $\begin{array}{l}\text { Não } \\
\text { Sim }\end{array}$ & $\begin{array}{l}33 \\
25\end{array}$ \\
\hline Secura vaginal & 2,576 & 0,109 & $\begin{array}{l}\text { Não } \\
\text { Sim }\end{array}$ & $\begin{array}{l}20 \\
38\end{array}$ \\
\hline Grauauto-estima & 0,008 & 0,930 & $\begin{array}{l}\text { Baixo } \\
\text { Bom }\end{array}$ & $\begin{array}{l}15 \\
43 \\
\end{array}$ \\
\hline Tempo de menopausa & 0,142 & 0,706 & $\begin{array}{l}<=\text { média } \\
>\text { média }\end{array}$ & $\begin{array}{l}39 \\
19 \\
\end{array}$ \\
\hline Idade $1^{0}$ orgasmo & 2,656 & 0,103 & $\begin{array}{c}<21 \text { anos } \\
>=21 \text { anos }\end{array}$ & $\begin{array}{l}32 \\
26 \\
\end{array}$ \\
\hline Válidos & & & & 58 \\
\hline Excluídos & & & & 2 \\
\hline Total & & & & 60 \\
\hline
\end{tabular}

A Tabela 2 apresenta o modelo de Regressão Logística elaborado para avaliar a influência das 17 variáveis independentes no CCO. Esta tabela apresenta também a estatística do teste para cada variável do modelo do CCO.
O teste de significância de ajuste do modelo estatístico contendo todas as variáveis exposto na Tabela 2 - teve como estatística o valor $c^{2}=38,615$ e $p=0,003$, significativo em nível de $5 \%$. Os níveis das variáveis são também encontrados na Tabela 2.
É importante salientar que o modelo contém variáveis que não contribuíram significativamente ao comportamento do CCO das mulheres. As variáveis não significativas foram retiradas do modelo de maneira iterativa, até que todas fossem significativas. $\bigcirc$ resultado do ajuste das variáveis no modelo final (modelo reduzido) para o CCO é apresentado na Tabela 3.

A Tabela 4 apresenta a comparação de previsões do modelo ajustado (completo) aos dados obtidos em relação ao CCO. A porcentagem de acerto nas previsões do CCO foi igual a $81 \%$ no modelo completo.

A Tabela 3 apresenta 0 teste de significância da contribuição das variáveis no modelo resumido; o valor de $p<0,050$ indica que a variável tem correlação positiva (ou seja, é significativa) com o CCO, considerando-se as demais variáveis conjuntamente. Ao final do processo iterativo de retirada do modelo completo das variáveis não significativas, observamos o valor de $c^{2}=32,762$ e $p<0,00$ I, indicando a significância do ajuste.

É importante salientar que as variáveis apresentadas na Tabela 3 foram analisadas conjuntamente, uma vez que as análises individuais podem não detectar as significâncias encontradas neste tipo de abordagem, devido a fatores de confusão (como as variáveis associadas tanto ao CCO quanto à variável que está sendo estudada).

$\mathrm{Na}$ Tabela 5, pode-se observar que, embora com número menor de variáveis, a porcentagem de acertos do modelo reduzido foi muito próxima à do modelo completo (Tabela 4). Este fato mostra que a exclusão de algumas variáveis não interferiu no nível de significância do teste.

A Tabela 3 apresenta também a distribuição conjunta das variáveis com contribuiç̧ão significativa. Esta apresentação tem o objetivo de facilitar a identificação do sentido de influência de cada variável no CCO. Observa-se que o CCO foi maior ou igual a um para $88,9 \%$ das mulheres que se masturbavam e para $30 \%$ das que não praticavam o auto-erotismo (Tabela 3).

O parâmetro "gostar de abraçar e acariciar o corpo do parceiro" associou-se positivamente ao CCO maior ou igual a um. A porcentagem de CCO maior ou igual a um foi também maior entre as mulheres com queixa de secura vaginal $(55,3 \%)$ do que entre aquelas que não apresentavam o sintoma (35\%) (Tabela3). 


\begin{tabular}{|c|c|c|c|c|c|c|c|c|}
\hline \multirow[b]{3}{*}{ Variáveisindependentes } & \multirow[b]{3}{*}{$p$} & \multirow[b]{3}{*}{ Qui-quadrado } & \multirow[b]{3}{*}{ Níveis } & \multicolumn{5}{|c|}{ Coeficiente de capacidade orgástica } \\
\hline & & & & \multicolumn{2}{|c|}{$<1$} & \multicolumn{2}{|c|}{$>=1$} & \multirow{2}{*}{$\frac{\text { Total }}{n}$} \\
\hline & & & & $n$ & $\%$ & $n$ & $\%$ & \\
\hline Prática da masturbação & $\overline{0,000}$ & 21,123 & $\begin{array}{l}\text { Não } \\
\text { Sim } \\
\text { Total }\end{array}$ & $\begin{array}{c}28 \\
2 \\
30\end{array}$ & $\begin{array}{l}\overline{70,0} \\
11,1 \\
51,7\end{array}$ & $\begin{array}{l}12 \\
16 \\
28\end{array}$ & $\begin{array}{l}\overline{30,0} \\
88,9 \\
48,3\end{array}$ & $\begin{array}{l}40 \\
18 \\
58\end{array}$ \\
\hline $\begin{array}{l}\text { Gostarabraçar/ } \\
\text { acariciar corpo parceiro }\end{array}$ & 0,036 & 4,392 & $\begin{array}{l}\text { Não } \\
\text { Sim } \\
\text { Total }\end{array}$ & $\begin{array}{l}17 \\
13 \\
30\end{array}$ & $\begin{array}{l}63,0 \\
41,9 \\
51,7\end{array}$ & $\begin{array}{l}10 \\
18 \\
28\end{array}$ & $\begin{array}{l}37,0 \\
58,1 \\
48,3\end{array}$ & $\begin{array}{l}27 \\
31 \\
58\end{array}$ \\
\hline Secura vaginal & 0,021 & 5,292 & $\begin{array}{l}\text { Não } \\
\text { Sim } \\
\text { Total }\end{array}$ & $\begin{array}{l}13 \\
17 \\
30\end{array}$ & $\begin{array}{l}65,0 \\
44,7 \\
51,7\end{array}$ & $\begin{array}{c}7 \\
21 \\
28\end{array}$ & $\begin{array}{l}35,0 \\
55,3 \\
48,3\end{array}$ & $\begin{array}{l}20 \\
38 \\
58\end{array}$ \\
\hline 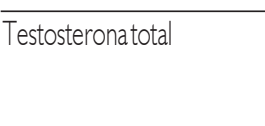 & 0,067 & 3,345 & $\begin{array}{c}<25 \\
>=25 \\
\text { Total }\end{array}$ & $\begin{array}{c}23 \\
7 \\
30\end{array}$ & $\begin{array}{l}59,0 \\
36,8 \\
51,7\end{array}$ & $\begin{array}{l}16 \\
12 \\
28\end{array}$ & $\begin{array}{l}41,0 \\
63,2 \\
48,3\end{array}$ & $\begin{array}{l}39 \\
19 \\
58\end{array}$ \\
\hline
\end{tabular}

Tabela 4-Classificação baseada no modelo estatístico completo do coeficiente de capacidade orgástica - formado por todas as variáveisindependentes estudadas - enosvalores observados para o coeficientede capacidade orgástica

\begin{tabular}{cccc}
\hline & \multicolumn{3}{c}{ Previsão modelo completo } \\
\cline { 2 - 4 } Observado & $<1$ & $\frac{>=1}{6}$ & $\frac{\% \text { correta }}{80,0 \%}$ \\
\cline { 2 - 4 }$>1$ & 24 & 23 & $82,1 \%$ \\
$>=1$ & 5 & $50,0 \%$ & $81,0 \%$ \\
\hline geral & $50,0 \%$ & & 5
\end{tabular}

\begin{tabular}{|c|c|c|c|}
\hline & & & \\
\hline Observado & $<1$ & $>=1$ & $\%$ correta \\
\hline$<1$ & 25 & 7 & $78,1 \%$ \\
\hline$>=1$ & 6 & 22 & $78,6 \%$ \\
\hline \%geral & $51,7 \%$ & $48,3 \%$ & $78,3 \%$ \\
\hline
\end{tabular}

A variável dosagem de testosterona total foi mantida no modelo reduzido (Tabela 3) apesar de não ter sido significativa no nível fixado para o estudo (5\%), porque há indícios de que ela influencie significativamente o CCO. Dentre as mulheres que apresentavam dosagens de testosterona total maior ou igual a $25 \mathrm{ng} / \mathrm{dl}, 63,2 \%$ apresentavam CCO maior ou igual a um. No grupo com dosagens de testosterona total menor que $25 \mathrm{ng} / \mathrm{dl}$ essa porcentagem diminuiu para $41 \%$. sentiam satisfeitas após a obtenção de dois orgasmos, e a terceira freqüentemente continuava a sentir desejo erótico após duas descargas orgásticas, atingindo até três orgasmos em uma só relação sexual.

Vinte e uma mulheres (35\%) alcançavam esporadicamente o orgasmo durante o encontro com o parceiro sexual e outras 21 (35\%) nunca obtinham orgasmo na presença do parceiro. Dentre estas últimas, duas mulheres (3,3\%) tinham anorgasmia primária, ou seja, nunca haviam experimentado o orgasmo e as outras $19(31,7 \%)$, apesar de terem sido orgásticas ao coito anteriormente, no momento da entrevista se submetiam à relação sexual sem obter a estimulação necessária para a descarga orgástica.

Ao estudar a prevalência de disfunções sexuais femininas nos Estados Unidos da América, Laumann et al. ${ }^{14}$ mostraram que cerca de $25 \%$ das mulheres não têm orgasmo. No Brasil, Abdo et al. ${ }^{15}$ observaram que $29,3 \%$ da população feminina queixam-se de dificuldades para obter orgasmo. Em nossa amostra, incluindo-se as mulheres com anorgasmia primária e secundária, encontramos 35\% de mulheres anorgásticas.

É importante, no entanto, salientar que a ausência total ou parcial de orgasmos na atividade sexual não constitui elemento suficiente para o diagnóstico de disfunção sexual. A Classificcação Internacional de Doenças, em sua décima revisão (CID- I0) ${ }^{16}$ lanç̧ada pela Orģanização Mundial de Saúde, define disfunção sexual como "os vários modos em que um indivíduo é incapaz de participar de uma relação sexual como ele/ela gostaria", observando-se, portanto, a necessidade do sofrimento subjetivo como critério para se definir a disfunção sexual.

Outro fato a ser salientado é que nosso estudo não avalia a incidência de disfunções sexuais, uma vez que a amostra estudada não representa a comunidade, mas constitui um grupo de mulheres saudáveis na pós-menopausa.

Atribuímos grande importância ao CCO, enquanto instrumento de avaliação da sexualidade, devido à sua eficácia em apontar se a mulher se expõe ou não à estimulação genital junto a seu parceiro, sem alcançar suficiente estímulo para a produção do orgasmo e sem compensar essa deficiência pela masturbação solitária. 
Este tipo de abordagem da sexualidade expõe aspectos ainda não estudados até 0 momento e revela características importantes de mulheres, na pós-menopausa, que apresentam equivalência entre a freqüência sexual e a freqüência orgástica. De acordo com as análises estatísticas, as mulheres que obtêm essa equivalência são aquelas que praticam a masturbação $(p=0)$, gostam de abraçar e acariciar $o$ corpo do parceiro $(p=0,036)$ e apresentam queixa de secura vaginal $(p=0,02$ I) (Tabela 3).

No momento da entrevista, $88,9 \%$ das mulheres que praticavam a masturbação apresentavam freqüência orgástica mensa/(número de orgasmos obtidos, por mês, durante a relação sexual e/ou a masturbação solitária) igual ou maior à freqüência sexualmensal, comparado a somente $30 \%$ das que não praticavam o auto-erotismo (Tabela 3). Esse resultado mostra que a masturbação constitui, efetivamente, uma forma de contrabalançar a dificuldade de se obter orgasmos com o parceiro.

A prática do auto-erotismo e a satisfação sexual foram estudadas em mulheres búlgaras por Leitenberg et al. ${ }^{17}$. Os autores não encontraram, no entanto, correlação entre a prática da masturbação na adolescência e pré-adolescência com a satisfação sexual de mulheres no início da idade adulta.

A Tabela 3 mostra que o grupo de mulheres com CCO $\geq$ I caracterizou-se por maior afetividade no relacionamento com o parceiro, denotada pelo fato de gostarem de abraçar e acariciar o corpo do mesmo $(p=0,036)$. No grupo de mulheres que não gostam do contato com o parceiro, 63\% delas apresentam CCO $<$ I, enquanto no grupo das mulheres que apreciam o contato essa porcentagem é de $41,9 \%$.

Os estudiosos da sexualidade correlacionam, freqüentemente, as características físicas do parceiro, seu desempenho sexual e o grau de afetividade do relacionamento com parâmetros sexuais femininos. Têm sido apontadas associações positivas entre a satisfação sexual e a atração física pelo parceiro, a intimidade, o amor e a satisfação com o relacionamento em geral, 78,19 .

Estudo sobre a prevalência de disfunções sexuais femininas nos Estados Unidos da América $^{14}$ mostrou que $20 \%$ das mulheres com idade de 18 a 59 anos apresentam dificuldades na lubrificação vaginal, sendo a queixa ainda mais comum em mulheres na pós-menopausa devido à redução do fluxo sangüíneo pélvico.

Nossos resultados mostram, no entanto, que as mulheres que apresentam secura vaginal, mas que praticam a masturbação solitária e mantêm relacionamento afetivo com o parceiro, conseguem obter o mesmo número ou um número maior de orgasmos se comparados à frequêencia do coito. A Tabela 3 mostra que, no grupo de mulheres com secura vaginal, 55,3\% delas apresentam freqüência orgástica - durante o coito e/ou a masturbação - maior do que a freqüência sexual (ou seja, CCO maior ou igual a um), enquanto no grupo das mulheres que não apresentam secura vaginal essa porcentagem é de $35 \%$.

A variável dosagem sérica de testosterona total foi preservada no modelo reduzido do CCO apesar de não ser significativa no nível fixado $(p=0,067)$, porque pareceu influenciar significativamente a capacidade orgástica (Tabela 3).

O CCO resultou maior ou igual a um em $63,2 \%$ das mulheres com dosagens séricas de testosterona total $\geq 25 \mathrm{ng} / \mathrm{dl}$ e em $41 \%$ daquelas com níveis séricos abaixo do valor de referência (Tabela 3). Kaplan" observou comprometimento da capacidade orgástica em mulheres com baixos níveis de testosterona sérica total e Grazziotin' ressaltou a importante ação dos androgênios no condicionamento da sensibilidade clitoridiana que, por sua vez, favorece a capacidade orgástica.

As pesquisas na área da sexualidade encontram-se em estágio inicial, sendo necessários estudos anatômicos que aprofundem o conhecimento da fisiologia da resposta sexual feminina. Investigações acerca do papel dos agentes vasoativos e dos esteróides sexuais na sexualidade feminina são também necessárias.

\section{Conclusões}

I) Nas mulheres pós-menopausadas avaliadas, a capacidade orgástica relacionou-se positivamente com o relacionamento mais afetivo com o companheiro e com a prática da masturbação;

2) A capacidade orgástica não foi comprometida nas mulheres com secura vaginal que praticavam a masturbação e mantinham relacionamento afetivo com o companheiro.

Conflito de interesse: não há.

\section{SUMMARY \\ ASSESSMENT OF ORGASMIC CAPACITY OF POSTMENOPAUSAL WOMEN}

OBJECTNE. To correlate the capacity of healthy postmenopausa/women to have orgasms (during intercourse or by solitary masturbation) with psychosocial, behavioral, climacteric, hormonaland interpersonal factors.

METHODS. Nine hundred and ninety-nine women (aged from 41 to 60 years) underwent physical and supplementary tests and answered questionnaires regarding sexual behaviour. Sixty healthy women, sexually active, with one ormore years of amenorrhea, without hormone therapy and with a partner capable of intercourse were chosen from this group. A Logistic Regression Model with one dependent variable - orgasmic capacity - and seventeen independent variables - psychosocial, behavioral, interpersonal, climacteric and hormonal factors - was developed.

RESULTS. The orgasmic capacity correlated significantly with the practice of masturbation $(p=0.000)$, with pleasure in embracing and caressing the partner's body $(p=0.036)$ and with the presence of vaginaldryness $(p=0.021$ ).

CONCLUSIONS. This study shows that the most importantfactors were interpersonaland behavioral and that the other parameters considered were not statistically significant. Women with vaginal dryness, who however engage in masturbation and maintain an affective relationship with their partner, obtain an equal or a greater number of orgasms when compared to the frequency of sexual intercourse. [Rev Assoc Med Bras 2004; 50(4): 444-50]

KeY words: Post-menopause. Orgasm. Masturbation.

\section{REFERÊNCIAS}

1. Graziottin A. Libido: the biologic scenario. Maturitas 2000;34:9-16.

2. Sarrel PM. Effects of hormone replacement therapy on sexual psychophysiology and behavior in postmenopausal. J Womens Health Gender Based Med 2000;9:25-32.

3. Bancroft J. Human sexuality and its problems. Livingstone: Churchill; 1989.

4. Kinsey AC, Pomeroy WB, Martin CW. Sexual behavior in the human female. Philadelphia: WB Saunders; 1953.

5. Masters WH, Johnson VE. Human sexual response. Boston: Little Brown; 1966. 
6. Basson R, Berman J, Burnett A, Derogatis L. Report of the International Consensus Development Conference on female sexual dysfunction: definitions and classifications. J Urol 2000; 1 63: 1 88-93.

7. Penteado SRL. Avaliação da sexualidade em mulheres na pós-menopausa [dissertação] São Paulo: Faculdade de Medicina, Universidade de São Paulo; 2002

8. Abdo CHN, Moreira JR, Fittipaldi JAS. Estudo do comportamento sexual no Brasil - ECOS. Rev Bras Med 2000;57: 1329-35

9. Golombok S, Rust J. The Golombok and Rust inventory of sexual satisfaction. London: NFER-Nelson; 1986

10. Abraham GE. Radioimmunoassay of steroids in biological materials. Acta Endocrinol 1974; 183:42-7.

II. Kaplan HS. Disorders of sexual desire. New York: Simon and Schuster; 1979
12. Morrison DF. Multivariate statistical methods New York: McGraw-Hill; 1967.

13. McCALL RB. Fundamental statistics for psychology. Hardcourt: Brace \& World Inc; 1970.

14. Laumann EO, Park A, Rosen RC. Sexual dysfunction in the United States: prevalence and predictors. JAMA 1999;281:537-44

15. Abdo CHN, Oliveira WM, Moreira JRED, Fittipaldi JAS. Perfil sexual da população brasileira: resultados do estudo do comportamento sexual (ECOS) do Brasileiro. Rev Bras Med 2002:59:250-7

16. Organização Mundial de Saúde. Classificação de transtornos mentais e de comportamento da CID-10. Porto Alegre: Artes Médicas; 1993.

17. Leitenber GH, Detzer MJ, Srebnik D. Gender differences in masturbation and the relation of masturbation experience in preadolescence and/or early adolescence to sexual behavior and sexual adjustment in young adulthood. Arch Sex Behav 1993;22:87-98.

18. Lawrance K, Byers ES. Sexual satisfaction in heterosexual long-term relationships the interpersonal exchange model of sexual satisfaction. Pers Relat 1995:2:267-85

19. Young T, Luquis R. Sexual satisfaction among married women age 50 and older. Psychol Rep 2000;86: | 107-22.

Artigo recebido: 15/08/03 Aceito para publicação: 09/06/04

\section{www.ramb.org.br}

Em nosso site, você encontra as edições da Ramb on line com o mesmo conteúdo da Ramb impressa: as seções À beira do Leito, Comentários, Diretrizes em Foco, Panorama Internacional, Discussão de Caso, Imagem em Medicina, Prática Clínica e ainda artigos originais.

\section{Acesse www.ramb.org.br}

